\title{
POSSIBILITÉS D'AMÉLIORATION, PAR LA SÉLECTION, DE LA PRODUCTION FROMAGERE DE LA RACE PIE ROUge de L'EST (1)
}

\author{
par
}

P. AURIOL

La production fromagère - et plus spécialement celle du gruyère - revêt une très grande importance économique et sociale pour l'agriculture jurassienne : plus des $3 / 4$ du lait produit dans le département sont en effet destinés à la fabrication du fromage, et c'est seulement grâce à une production de qualité - le gruyère de Comté - que les éleveurs de cette région peuvent espérer obtenir un prix rémunérateur de leur lait.

C'est pourquoi il nous a semblé intéressant d'étudier la production des vaches Pie rouge de l'Est, non seulement pour la quantité et la richesse en matières grasses de leur lait (contrôle laitier classique), mais aussi pour ses " qualités fromagères ".

L'existence de la Station de Recherches laitières de Poligny, en pleine région à gruyère et la création, en décembre 1952, d'un Syndicat destiné à organiser la mise à l'essai des taurillons devant assurer la remonte du Centre d'insémination artificielle de la Coopérative d'élevage du Jura, nous ont permis de mettre sur pied un programme de travail dont les buts principaux sont les suivants :

$1^{\circ}$ Etudier dans quelle mesure et de quelle façon la composition du lait influe sur la quantité et la qualité du gruyère obtenu, ainsi que sur les accidents qui peuvent survenir au cours de la fabrication et de la conservation. L'ensemble de ces travaux doit permettre, notamment, de définir les qualités fromagères que doit posséder un bon lait à gruyère.

$2^{\circ}$ Etudier l'influence des facteurs physiologiques et du milieu qui agissent sur la production fromagère d'une vache.

$3^{0}$ Etudier le mécanisme de la transmission héréditaire des qualités fromagères.

En dehors de ce travail, nous suivons également la transmission des caractères de conformation, de fécondité, rusticité et longévité, caractères qui permettent aux aptitudes fromagères de s'extérioriser pleinement.

Mais la sélection de ces différents caractères nécessite auparavant l'étude et la mise au point de méthodes de contrôle simples et précises, qui nous permettront de suivre les variations de chacun d'eux.

(1) C. R. Acad. Agr., 1954, 8331. 
Enfin, il nous restera à déterminer les parts respectives que jouent le milieu et le potentiel héréditaires dans la manifestation de ces caractères.

\section{Qualités fromagères}

Les études poursuivies par la Station de Recherches laitières de 'Poligny et par la Station centrale de Microbiologie et de Recherches laitières [2] depuis plusieurs années déjà, nous conduisent à considérer le problème sous deux aspects, l'un quantitatif, l'autre qualitatif.

La production fromagère d'une vache dépend en effet :

- De la quantité de lait sécrétée ;

- De la teneur en matières azotées de ce lait (et surtout en caséine) ;

- De sa teneur en matières grasses.

Quant à la qualité du fromage obtenu, si elle est largement conditionnée par la valeur professionnelle du fromager et par la qualité bactériologique du lait à travailler, elle dépend aussi de la composition même du lait et, en particulier, des proportions qui existent entre les différents constituants organiques et minéraux.

A cet égard, le rapport entre le taux de calcium et celui des substances azotées présente certainement une grande importance et il est intéressant de sélectionner pour la fromagerie des laits dont le rapport $\mathrm{Ca} / \mathrm{N}$ soit élevé (MOCQUoT et collaborateurs).

Dans le cas d'un lait pauvre en calcium, on observe très souvent une mauvaise coagulation et des difficultés d'égouttage pendant la fabrication, difficultés qui se manifestent plus tard, au cours de l'affinage par le défaut, connu sous le nom de "lainure " (la pâte du fromage se fend). Les fromages lainés sont très dépréciés et ceci se traduit par des différences de prix parfois considérables au moment de la vente.

Le problème de la qualité est, nous l'avons dit, très important pour un produit tel que le gruyère de Comté et il est bon de rappeler qu'entre un fromage de premier choix et un fromage défectueux, la différence de prix peut aller du simple au double (de 250 à $450 \mathrm{fr}$. le kilogramme pour le premier semestre de 1953).

La durée de coagulation du lait par la présure, qui est d'ailleurs liée, dans une bonne mesure, à la valeur du rapport $\mathrm{Ca} / \mathrm{N}$, représente également un bon test d'appréciation des qualités du lait pour la fromagerie. On rencontre assez fréquemment des vaches produisant un lait anormal, qui ne caille pas ou très mal sous l'action de la présure. De tels laits doivent être éliminés et, si l'on veut assurer une bonne fabrication, on ne doit employer que des laits dont la 
vitesse de coagulation est à peu près la même et ne s'écarte pas d'une valeur moyenne que l'on a reconnue favorable à la fabrication.

En résumé, un bon lait à gruyère, en dehors des qualités indispensables de propreté, doit :

- Etre riche en caséine ;

- Contenir au moins 36 grammes de matière grasse par kilogramme;

- Etre facile à travailler, c'est-à-dire réagir normalement à la présure et présenter un rapport $\mathrm{Ca} / \mathrm{N}$ élevé.

C'est en fonction de ces différents critères que le contrôle fromager a été organisé dans le Jura, en collaboration avec le Syndicat de testage de ce département.

Voici, après quelques modifications, la méthode actuellement employée : le Syndicat de Contrôle laitier départemental se charge, en plus des opérations ordinaires du contrôle laitier, des prélèvements suivants : chaque mois, lors de son passage, le contrôleur laitier prélève, pour les vaches étudiées, un. échantillon du lait de la traite du soir $\left(150 \mathrm{~cm}^{3}\right)$ et un échantillon du lait de la traite du matin $\left(150 \mathrm{~cm}^{3}\right)$. Après addition de formol, les deux flacons, portant indication des pesées respectives de lait, sont envoyés par la poste à la Station de Poligny, qui effectue le mélange proportionnel du lait du soir et du matin.

Le test de la présure devant être effectué sur le lait frais, aussitôt après chaque traite, c'est au contrôleur laitier que cette tâche a été confiée : pour chaque vache, il prélève à chaque traite $11 \mathrm{~cm}^{3}$ de lait dans un tube à essai et, après avoir ramené ce lait à $35^{\circ} \mathrm{C}$., il ajoute $1 \mathrm{~cm}^{3}$ d'une solution de présure, dont le titre est fixé de telle sorte qu'un lait normal caille entre quatre et huit minutes. Le contrôleur relève les temps de prise du lait soir et matin. Cette méthode permet de suivre le lait de 8 vaches en trente minutes environ.

Actuellement, toutes les vaches inséminées par un taurillon en testage et qui donnent naissance à une génisse sont automatiquement soumises au contrôle fromager. Nous pensons atteindre le chiffre de 350 vaches contrôlées mensuellement dès le mois de septembre prochain.

Au cours de l'année 1953, la Station de Recherches de Poligny a analysé d'une façon plus détaillée le lait de 50 vaches en examinant également la composition du lactosérum. Cette analyse nécessitait la fabrication à la ferme d'un caillé à partir de $300 \mathrm{~cm}^{3}$ de lait et la récolte de $150 \mathrm{~cm}^{3}$ de lactosérum par traite, ce qui ne permettait plus de suivre plusieurs vaches dans la même étable, par suite 
du temps qu'exigeait cette opération. On s'est donc contenté, par la suite, de prélever seulement l'échantillon de lait.

Tous les échantillons mensuels, centralisés à la Station de Poligny, y subissent le dosage des matières azotées totales (méthode Kjeldahl), du calcium et de la matière grasse.

En dehors des analyses de laits individuels, nous avons entrepris, depuis mars 1953, un contrôle des laits d'étables et de fromageries, de façon à suivre la production aux différents stades : individu, troupeau, fromagerie. Ces analyses doivent, en particulier, nous permettre d'étudier les variations entre troupeau la même année et les variations d'une année sur l'autre dans le même troupeau, ce qui nous donnera la possibilité de chiffrer les variations annuelles dues aux différences de milieu.

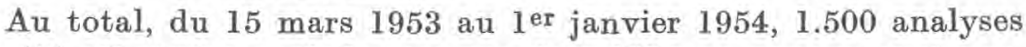
ont été effectuées, mais le manque de crédits ne nous permet pas, pour l'instant, de généraliser les contrôles d'étables et de fromageries, comme il serait souhaitable de le faire (il faudrait arriver à suivre au moins une fromagerie par grande région naturelle).

Les méthodes de contrôle mises au point, il reste à déterminer les causes des variations observées dans les résultats.

\section{Influence des facteurs physiologiques et du milieu sur la production fromagère individuelle}

En dehors du grand intérêt scientifique qu'il présente, l'examen de ces différents facteurs est nécessaire pour permettre une comparaison valable de la production des filles à celle de leurs mères, lors de l'interprétation des résultats du progeny-test. Nous aurons, en effet, rarement la chance d'avoir à comparer une mère et une fille ayant vêlé à la même époque, dans les mêmes conditions de milieu. De plus, très souvent, nous ne pourrons comparer la première lactation de la mère avec celle de sa fille.

Les variations du taux de matières azotées totales (diminution rapide pendant le $1^{\text {er }}$ mois de lactation, puis palier et remontée à partir du $4^{\mathrm{e}}$ ou $5^{\mathrm{e}}$ mois de gestation, JARRIGE, 1954) font que la courbe de production totale de matières azotées présente un profil un peu atténué par rapport à la courbe de lactation, mais sans en différer notablement : le principal facteur du rendement fromager reste la quantité de lait sécrétée. Ceci est d'autant plus vrai que l'âge et le numéro d'ordre de la lactation ne semblent pas avoir une grosse influence sur le taux de matières azotées du lait, comme le montrent les premiers résultats obtenus dans le Jura.

Nous sommes donc ramenés d'abord à l'étude de l'influence des facteurs physiologiques et du milieu sur la quantité de lait sécrétée et ceci dans les conditions particulières de l'élevage jurassien. 
Facteurs physiologiques. - Age au premier vêlage. - Ce facteur a une grosse influence sur la production, du moins au cours de la première lactation, et les filles des taureaux en testage étant jugées d'après leur première lactation, il est indispensable de le faire intervenir. De plus, cet âge est très variable, suivant les régions et surtout suivant les éleveurs.

Numéro d'ordre de la lactation et correction pour l'àge. - L'étude de l'évolution de la production laitière avec l'âge, déjà effectuée sur un certain nombre de races étrangères. n'a jamais été abordée sérieusement dans la race Pie rouge de l'Est. Il se peut du reste que nous soyons obligés de considérer séparément les deux rameaux Tacheté et Montbéliard, qui semblent présenter, à première vue, une évolution un peu différente.

De plus, l'augmentation avec l'âge de la quantité de lait produite serait moins importante pour les vaches qui ont débuté par une première lactation forte que pour les autres, et nous serons sans doute amenés à distinguer ces deux catégories pour établir deux tables de correction pour l'âge, suivant la production au premier vêlage.

Nous étudions, également, d'autres facteurs physiologiques, tels que l'intervalle vêlage-saillie, la période d'arrêt de la sécrétion lactée, facteurs qui, pour des vaches ayant un cycle anormal de reproduction, peuvent jouer un rôle important.

Facteurs du milieu. - Dans le Jura, pays essentiellement varié, ces facteurs viennent modifier de façon très différente les rendements des vaches, ainsi que leur comportement.

Nous suivons actuellement l'influence du mois de vêlage et celle de l'alimentation. Si la première est relativement facile à apprécier, la seconde exigerait des analyses généralisées de fourrage (herbe et foin), permettant de classer les étables en deux ou trois catégories suivant le niveau de l'alimentation.

\section{Transmission héréditaire des qualités fromagères}

L'étude de la transmission héréditaire des qualités fromagères n'a été abordée, à notre connaissance, que par un très petit nombre de chercheurs.

Différentes observations semblent cependant prouver qu'elles sont sous la dépendance étroite de facteurs génétiques: les travaux de Hancock [4], montrent que les jumelles univitellines ont des taux de caséine très voisins.

De plus, dans la même étable, il existe des différences très notables d'une vache à l'autre, au même titre que pour le taux butyreux.

Enfin, nous trouvons des différences radicales très nettes, qui 
sont mises en évidence par la comparaison des analyses effectuées par différents ehercheurs étrangers et par JARRIGE à la Station de Recherches sur l'Elevage du C.N.R.Z. [6]. La race Pie rouge de l'Est aurait, d'après les premiers échantillons analysés, un taux de matières azotées moyen de 30 à 32 grammes par kilogramme de lait, ce qui représente une valeur relativement faible par rapport aux autres races étudiées.

Mais dans quelle mesure les variations individuelles observées sont-elles causées par des différences dans la composition du potentiel héréditaire? L'étude d'un grand nombre de corrélations entre les productions d'individus parents (en particulier mères-filles dans le cas du progeny-test) nous permettra de déterminer la valeur de l'héritabilité de ces caractères. Pour Hancock [5], Bonnier et al. [1], cette valeur serait de l'ordre de 0,5, e'est-à-dire que $50 \%$ des variations observées dans les taux de matières azotées individuels seraient dus à des facteurs génétiques.

Par sélection, nous devons donc pouvoir obtenir des vaches grandes fromagères, comme on a pu obtenir des vaches grandes beurrières. Le testage entrepris dans le Jura doit mettre en évidence des taureaux transmettant régulièrement à leurs filles de bonnes qualités fromagères.

Un autre aspect très important du problème de la sélection fromagère est le suivant : les vaches donnant un lait riche en matière grasse sércètent-elles aussi, obligatoirement, un lait riche en caséine?

De nombreux calculs de corrélation entre taux de matières azotées et taux de matière grasse sembleraient montrer qu'il y a effectivement une corrélation nettement positive entre les deux. mais ces études portent le plus souvent sur des laits de mélange, Par contre, lorsqu'il s'agit de laits individuels, un certain nombre d'auteurs étrangers : Lonka [8], Larsson [7], GaInes [3] st JARRIGE [6], rapportent l'existence de grosses différences entre teneur en matière grasse et teneur en matières azotées du lait chez un certain nombre d'individus; nous-mêmes, dans le Jura, avons retrouvé des différences du même ordre.

Il n'est done pas possible de se contenter d'une analyse de la matière grasse pour apprécier la richesse du lait en caséine, du moins pour un travail de sélection. Il est indispensable d'envisager, pour l'avenir, la mise au point d'une technique simple et rapide de dosage des matières azotées du lait, technique qui devra pouvoir être utilisée sur place par les contrôleurs laitiers, en même temps que le contrôle laitier ordinaire.

Nous concluerons, avee E. L. Larsson [7], qu'il existe, en plus des variations héréditaires de la teneur du lait en matières azotées 
liées à la variation héréditaire de sa teneur en matière grasse, une variation héréditaire considérable de la teneur en matières azotées, indépendante de celle de la teneur en matière grasse et suffisamment grande pour qu'on puisse envisager une amélioration des qualités fromagères par sélection.

\section{$* * *$}

Signalons enfin, pour être complets, que cette sélection fromagère entre dans un plan beaucoup plus général de sélection du bétail laitier du Jura, plan dont les objectifs principaux peuvent être résumés de la façon suivante :

- Production fromagère maximum ;

- Bonne conformation (surtout aplombs et mamelle) ;

- Rusticité, fertilité et longévité.

Les avantages consentis aux éleveurs participant aux opérations de testage (insémination artificielle gratuite) ont contribué largement à développer le contrôle laitier dans cette région (une centaine d'adhérents nouveaux au cours de l'année 1953), ainsi que les inscriptions aux différents herd-books.

Nul doute que le testage entrepris dans le Jura, en dehors de son intérêt scientifique et technique, ne représente une occasion de coopération fructueuse entre les éleveurs, les différents services officiels intéressés et les organismes d'élevage, ainsi qu'un moyen de vulgarisation puissant.

\section{(Station de Recherches sur l'Elevage I.N.R.A.)}

\section{BIBLIOGRAPHIE}

[1] G. Bonnier et A. Hansson. Acta Agric. Suecana, 1946, 2, 171-184.

[2] R. Chevalier, G. Mocquot, C. Alais et M. Bonnet. Compte rendu Acad. Sc., 1950, 230, 581-231, 249.

[3] W. L. Gaines. Cité par Lonka [8].

[4] J. Hancock. N.Z.J. Sci. Technology, 1950, sec. A, 31, 5.

[5] J. Hancock. M.Z.J. Sci. Technology, 1953, sec. A, 35, 2.

[6] R. JARRIGE. (Sous presse).

[7] E. L. Larssow. Mejeritekniska Meddelanden, 1950, 11, 14.

[8] T. LonkA. Maataloustieteellinen Aikakauskirja, 1947, 19. 